\title{
Study on Image Design in Animation
}

\author{
Minghua Liu \& Ping Wang \\ Art School of Northwest University \\ Xi'an 710069, Shaanxi, China \\ E-mail: tangwind@gmail.com
}

\begin{abstract}
In this article, the author makes analysis and study on the three aspects of the technique of expression of image design in animation, artistic style of its image design and local characteristics of its image design, and analyzes such figures of speeches as transformation, exaggeration, combination and personification. It is exactly these expressive elements that enrich image of animation and fills it with more vitality. This article mainly studies the expressive elements of image design from the three perspectives of artistic style, that is, modeling style, motion performance, and indisguise, while design of modeling, motion and indisguise are expressive elements without which the style of animation can not go. Finally, the author explores and studies local characteristics of Chinese animation, Japanese animation and American animation, and comes to the conclusion that image design of animation has indiscerptible relationship with local characteristics of a country and local cultural tradition of the country has a direct effect on design connotation of the image.
\end{abstract}

Keywords: Cartoon, Image design

\section{Introduction}

Animation image is the most characteristic animation element, and thus has the most expressive force. Design of the image of characters in animation is to demonstrate the internal spiritual ethos and traits of character through their external image. Quality of the image design of animation has a direct effect on vividness of the entire animation, and furthermore, influences the immersion feeling of audience. Design of image of animation plays an immeasurable role in development of animation.

\section{Technique of expression in the image design of animation}

There are some companies that still employ traditional animation methods in particular situations. At present, some animation manufacturers employ the method of frame, which realizes TF Tween Frame by use of computer. This method is mainly used in two dimensional animations. Another method is virtual technology, which is used in three dimensional software, and is mainly applied in three dimensional animation, realized by Maya or 3Dmax. Besides, there is also the computer motion capture technology, which is directly performed by real people, and which is transferred to animation after capture of motion by the computer. The final one is immediate generation technology, which is mainly applied in game playing. Its basic principle is to separate expression, motion and changes of characters and objects into several subsections to draw multiple pictures, and finally to shoot continuously these pictures into a series of pictures with a virtual camera, producing a continuously changing effect to the vision. Animation is a sort of visual artistic form which is humorous, active in its technique of expression and has somewhat the implication of caricature. Its image design methods should follow the artistic form and artistic characteristics of design.

Image design of animation is not only a symbol of the image, but also has some other properties, such as external appearance of the image, its characteristics and motion, etc. Only if these properties are endowed to the image of design, can a designer succeed in designing a lifelike and vigorous image. Image design is a fruit of the rich imagination of an animation designer who employees several figures of speech, such as exaggeration, transformation, combination and personification, etc, to bring life to the lifeless organism, to abstract the image of concrete versachlichung, to endow animals and plants with humane characters, to bring people into integration of illusion and reality, and to delineate vivid, interesting, peculiar and humorous visual image, which will bring strong visual impact to human beings and leave them with a deep impression. Although there are several methods for the image design of animation, they can be mainly generalized as the following several:

\subsection{Exaggeration and transformation}

Exaggeration is to exaggerate a certain motion and form of a role to emphasize characteristics of an image based 
on the reality of the visual object in the objective world. In the image design, it is the distinct characteristics of the position that is mostly exaggerated, making the long part longer, the short part shorter, the fat part fatter and the thin part thinner, etc, which may make the form and image of a role more vivid and its appearance characteristics more distinct and prominent. For instance, at the earlier stage in China, the motion picture film mainly adopted the means of folk art for fabrication, and the paper-cut, puppet, clay animation and shadow puppet, etc, employed the technique of expression of exaggeration and transformation to endow the image of characters and animals with more clear-cut characteristics. There has been someone who says, "Let's have a look at the method of Disney in its modeling of professional characters: after the modeling designer has an in-depth grasp of the drama, he will then search for a model with similar character with the professional character, and copy the model from the real life. After having seized the overall shape and characteristics of the target, the designer then abstracts and refines him/her. Only such a role created can impress the audience. It thus is obvious that, transformation and exaggeration both are two important technique of expression, and one should not be partial to either of the two.

\subsection{Combination}

Combination is a frequently used suppositive image design method, in which a designer organizes different objects into new image after his processing. The designer fabricates those imaginary objects that may not exist in the real life after combination or confabulation so as to create the image of animation that people may not have a chance to see or that may not exist in the objective world. This highly suppositive combination method in animation is one of the important factors for animation arts to absorb people's vision. In the motion picture film, some images can only be described by words in the fairy tale, and there are some supernatural beings and demons and ghosts that do not exist in the real life and some characters in the science fiction world, all of which can be performed as large as life in the screen and leaves an unforgettable impression on human beings. The images of roles in "J Monsters Inc" and "Curse of the Devil" do not exist in the objective world, and these images combine characteristics of images of all sorts of objects and design each distinct and weird role. Carl once said, "If one intends to become a top-level animator, he/she has to be an excellent artisan-painter." Animation drawing is not to follow suit, and it requires understanding and expression. Different from the actual persons and objects in film-shooting, the image and motion in animation are imagery materialization forms by artists and are created artificially. Therefore, according to the subjective will of human being, different personalities and modeling combinations can be expressed in a suppositive way. This is also the reason why animation is suitable for the subject matters of fairy tales as a peculiar audio and visual language.

\subsection{Personification}

The designer conducts "personification" on the target of his creation, and endows it with emotional color. The method of personification is most prominent in the expression of animation art. Looking back at the various lively cartoon images in the animation art, it seems that the alive and kicking characters in our real life are, as a matter of fact, humanization of cartoon image. Personification mainly refers to the fact that characters and personality of human beings are endowed to those targets without any thinking or feeling, which may personalize them. The three guinea pigs, one star-nosed mole and one fly in the G-Force all have their own advantages and are willing to dedicate themselves to their own occupation, with distinct, kind-hearted, optimistic, innocent and naughty "humane" characters. Furthermore, the brave, stubborn and persistent animation image of Wall-E in the film "WALL-E." is lovely.

\section{Artistic style of image design in animation}

\subsection{The modeling style of image design}

The image of a role which has the image of an animation in an animated film may be a character, an animal or a plant, and after being processed by the image design in an animation, the well-known object can be transferred into a totally strange audio and visual image beyond expectation of the public. Such an image can absorb curiosity of the audience, and enable them to enjoy the great surprise of visual art at the time of enjoying happiness brought by the animation. For example, in "Hauru no ugoku shiro", when the creator in chief make the modeling design, he also makes corresponding corrections on the basis of the fairy tale. When he implants new vivacious life to the castle, he adds the mischievous image of "frame" that does the cooking, which makes the audience unforgettable. In addition, different types of modeling for animation roles can also present and convey different national temperament and interest. For instance, in the classical animation film "Avanti's Story", the traditional design element of puppet was used to express the plot. This film modeled successfully a legendary figure with distinct characteristics through exaggerated figure image, humorous dialogue and design of a goatee that was characterized by his intelligence, wisdom, abhorring evil as a deadly foe, his hating injustice and his 
humor, etc. The film concentrated very much on design of the color and the transition of the softness in its design, and it used such colors as cyan, green, red, white, blank and yellow which were commonly used among the common people. In its design of the image, it paid special attention to the undemonstrative goatee of Avanti, his aquiline nose, his small round eyes with the eyebrow of a willow pattern, the "Selan" cloth twined on his head, his little cap, the plucked instrument in his hand, his riding on a little donkey travelling around, and his contracted outline, which brought a fresh feeling to the audience, and which, at the same time, was full of the lingering charm of traditional art.

\subsection{The motion expression of the image design}

The design of motion in animation refers to design of the movement state of a role in animation, which includes positioning of characters of an animation image and positioning of the motion characteristics, etc. Motion design must be the most characteristic pattern positioning according to different movement processes of an animation image to endow the character of each image with rational embodiment. Humor and drama are the major characteristics of the motion design in an animation image, whereas performance is the compulsory course in the motion design in an animation. The performance art itself is the classical and sublimated realistic life, but not the original state of life. The motion design of an animation is also an attempt to highlight and emphasize this major point, so it has more aesthetic meaning. The style of motion design is closely bound up with the style of image design. If the style of the image design is exaggeration, then correspondingly, the style of the motion design is also exaggeration. If the style of the image design is the expressive method of a caricature, then the style of the motion design should also be the expressive method of caricature. Some extremely exaggerated motion design may bring people absurd, weird and inconceivable feeling, but it also corresponds with the illusion or subconscious image pattern of the audience. Thus, such an exaggerated and humorous performance and motion design is in line with the rule of artistic creation, and is also in line with the rule of the nature. It may be quite clear to the audience that this is just to express particular sentiment, feelings and ideas, and is to magnify a certain familiar expression into a special visual symbol. Such a symbol may bring people a newfangled visual and psychological feeling, stimulus or excitement, etc, totally different from the previous visual experience. This diverse visual feeling is exactly the charm of animation and, moreover, is the purpose pursued by the motion design in animation. As is well known, the image of animation is diversified, with characters, animals and all sorts of living beings, and in most cases, some props may be designed into animated objects. Thus, the movement rule of the motion design seems to be extremely important. Since different animation images have different structures, the rule and rhythm of movement are also different. For instance, the "Kung Fu Panda", an outcome combined by the traditional Chinese culture and advanced American technology, can be said to be a combination of Chinese and Western elements. The lively Panda A Bao, its leaping onto roofs and vaulting over walls, its siddhi of hitting at certain acupoints, and styles, schools, swordsman spirit and code of the brotherhood that are common in the gongfu stories of all sorts, and the framework of the novels by Jin Yong and Gu Long, and even the thinking of the Buddhism, are all be reflected in the film. The audience can follow the rhythm of the picture and the mellifluence of the motion of the role to experience interest and aesthetic feeling of the movement, and obtain the visual aesthetic feeling. Furthermore, for the role of the general in "Pride General", it borrows the facebook and movement in a Beijing opera, and is matched up with rhythmed traditional Chinese opera drumbeat, which makes the movement of the role novel and elegant, full of traditional Chinese cultural characteristics.

\subsection{The hypothetical artistic style}

Animation is to exploit the temporary residence of the visual sense of human being to cause a hypothetical phenomenon and the process of the entire animation is pictures of some static, continuous and rapid projection. For instance, in the case of the Disney animation, it is characterized by the following: it is centered by the feature film, sinuous in plots, distinct in characteristics of figures, vivid and exaggerated in performance of movement, exquisite and pleasant of the music, and suitable for the aesthetic taste of a large majority of the audience. Starting from the animation film "Snow White", Disney extensively draws materials from classical stories and recomposed many fairy tales, fables and folk legends into animated films, such as, "Cinderella", "Alice In Wonderland", "Sleeping Beauty", and "The Adventures of Pinocchio", etc. All these classical animation films have received extensive favor from children around the world. The most fundamental feature of animation art is its assumption, and is a film art with high assumption. It can be said that, there is not any other visual art that has more imagination or surprise power than animation. The latter can turn time back and twist the space, in which a designer can express his own ideas without any obstruction in an unfettered way, so people may enjoy this particular artistic charm to the top of their bent in the process of watching a film. Although this artistic expression of assumption exists in any art, it has great significance to animation art, because either macro 
animation art or concrete animation image can not go without assumption. Assumed occasion, assumed prop and assumed image are all developed together with assumed plots. Assuming that we do away with assumption of animation, obviously there would be no classic and mystical Son Goku, the monster Shrek the Third who is ugly but attracted toward freedom and love, or the time of brutality filled with surprise and danger --- "Ice Age", in which a precipitated abandoned baby enabled three totally disparate animals to get together. The caustic mammoth, the barbaric, impolite and giant sloth and the guileful eusmilus, the three prehistoric animals not only have to be the baby-sitters of the small baby, but have to go through the dangers of glacier and iceberg to protect it back home. Thus, it can be seen that, assumption of the image of an animation role not only adds more aesthetic characteristics to creation of animation art, but also brings unexpected artistic effect and visual and audio enjoyment to the audience.

\section{Local characteristics of the image design in animation}

Considering the art design styles in numerous Chinese and western animation films, animation artists of all nations put their hands to local traditional artistic forms and absorb quintessence of indigenous culture and particular artistic manifestation means to create animation artistic styles of their own and to continuously enrich their animation artistic styles and forms.

\subsection{Local characteristics of Chinese animation}

After undergoing cultural accumulation and inheritance for thousands of years, the traditional Chinese fine arts have formed rich and unique expressive system with local characteristics, which deserves further research and discussion by current animation creators. Expressive forms of the traditional Chinese fine arts include all sorts of traditional Chinese operas, relevant masks and costumes, all sorts of consummate and excellent technology, such as ceramic craft, knitting, knitting and dyeing, embroider, puppet, types of facial makeup, sculpture, bamboo \& rattan knitting, dough figurine fabrication, lacquer painting, toy fabrication and scissor-cut, etc. Scissor-cut, shadow puppet and new year picture are plane expressive art forms of two dimensions, which have concise but not stiff modeling, exaggerated but not monstrous, full of changes in unification, bold and exaggerated in image, and humorous but not constrained to realistic situation. The audience may experience the feeling and thinking of creators in clear and explicit composition and modeling. For example, the film "Avanti's Story" borrowed the traditional Chinese design element --- puppet--- for expression. In this film, the creator employed wire-pull or straining beam as a pull, with various lifelike forms, vivid and interesting patterns, extremely exquisite conception, including integration of several modeling arts, such as scissor-cut, industrial arts and painting, etc. All these traditional artistic forms can be rationally applied into image design of animation.

Individualized expressive language and expressive painting form in Chinese animation art design are quite rich, such as painting with exact delineation and enriched colours in single-Ping Tu, Chinese ink animation in lacquer painting, children's drawing, doodle, caricature, petrogram and wall painting all have a style of their own. Local culture establishes a kind of harmonious cultural ecological relationship, which is exactly the resource to be borrowed by modern animation artistic language and the soil it is going to reply on. For instance, the films "Golden Conch" and "Mr. Pig eats watermelon" adopt the expressive forms of Chinese shadow puppet and scissor-cut. These animation films express traditional Chinese folk arts as being bright and colorful, with strong nationalistic style. The works "Pride General" expresses the national feature and finds a path to bring into full play its peculiar national style in terms of characteristics of the characters and expression of motion and language, etc, which does not only correspond with the appreciation habits and aesthetic requirements of the public, but also enriches the treasure house of the world culture, widely welcomed and favorably received by the public. In terms of the forms of painting, in addition to traditional forms of oil painting, sketch, watercolour and crayon, such materials as metal, paper, mud, stem leaves of plants, plastics, rubber, glass and skin and hair of animals are all used for animation. The diversification of the design style of fine arts contains excellent elements of traditional Chinese culture.

\subsection{Local characteristics of American animation}

In the current world, American animation films also account for a large portion of the global animation market. The characteristics of American animation image are as follows: careful painting of the scene, which is enough for requirement of the plot. Usually, the image design of characters is excellent in modeling, distinct in personification, whereas under the new technology, roles of animation are more life-like, which attain perfect visual effect. "Ice Age" is reproduction of an already perfect works. Local design style of animation in America has an effect on creation thought and modeling style of animation design in quite a lot of nations in the world. 


\subsection{Local characteristics of Japanese animation}

Compared with heroism in American animation works, Japanese animation works focus more on the small and exquisite characteristics of the works, and furthermore, the meticulous Japanese culture is also the peculiarity of Japanese animation. Generally speaking, the characteristics of Japanese animation can be generalized as follows: exquisite painting, meticulous role, modeling and scene design, resorting to hyper-reality imagination space to satisfy illusion of the audience to realize what is impossible, and impaling the imaginary nerve of the audience. In the aspect of character roles, most heroes have a fine figure of a man, handsome, and most heroines are with long hair, oval face, exaggerated big eyes and a similarly exaggerated small nose, as well as a beautiful build. For example, considering all aspects of life, such as Japanese costume, food and drink, tea-making, product design and performance art, the film "Doraemon" can reflect the fact that Japanese people concentrate on exquisite and meticulous culture, which, in turn, is reflected in creation of its animation. Having been popular for several decades, this film can well reflect such an issue that is still widely and well received. This works mainly narrates a machinery cat called Doraemon who comes from the $22^{\text {nd }}$ Century and, entrusted by its master, employs a lot of mysterious props in the future to help its master Mahavira back in the $20^{\text {th }}$ Century.

\section{Conclusions}

All in all, the author makes an analysis and study on the expressive method of image design in animation, the artistic style of the image design and local characteristics of the image design, and analyzes the figures of speech of transformation, exaggeration, combination and personification. It is exactly these expressive elements that enrich the image of animation to a great extent and endows it with more vitality. In terms of artistic style, the author mainly studies expressive elements of artistic style of the image design from three aspects, namely, modeling style, motion manifestation and hypothetical artistic style, and modeling, motion and hypothetical design are three expressive elements that animation style can not go without. Then, the author conducts a comparative study on local characteristics of Chinese animation, Japanese animation and American animation. Different regional cultural characteristics bring about differences in the images of animation. In tue future, similar research should pay more attention to more design inspiration brought to the designer by such regional cultural differences, so that more local animation images can be generated in the country.

\section{References}

Chris Webster. (2009). Animation: the Mechanics of Motion. Beijing: Posts \& Telecom Press, September.

Fang, Jianguo, Wang, Peide \& Peng, Yi. (2007). The World History of Animation. Zhejiang: Zhejiang University Press, March.

Fang, Xiaoxi. (2008). Character Design of Animation. Beijing: Printing Industry Press, September.

Furniss, Maureen. (2009). International classical animation design course, translated by Fang, Li \& Li, Liang. Beijing: China Youth Press, September.

Qiao, Jingjing \& Lu, Hong. (2009). Creation of Cartoon. Shanghai: Shanghai People's Fine Arts Publishing House, June. 